\title{
Inclusive Standard Model Higgs searches with ATLAS
}

\author{
Francesco Polci
}

\author{
LAL, Univ. Paris-Sud, CNRS/IN2P3, Orsay, France
}

\begin{abstract}
The update of the discovery potential for a Standard Model Higgs boson through the inclusive searches $H \rightarrow \gamma \gamma$, $H \rightarrow Z Z^{*}$ and $H \rightarrow W W$ with the ATLAS detector is reported. The analysis are based on the most recent available simulations of signal, backgrounds as well as the detector response.
\end{abstract}

Keywords: Higgs, ATLAS

\section{INTRODUCTION}

The Higgs boson is the neutral scalar particle which breaks the electroweak symmetry of the Standard Model (SM) [1]. It is responsible for the mass of all other particles and it is the only one within the SM which has not yet been discovered. Theoretical arguments asses that the Higgs should have a mass below $1 \mathrm{TeV} / \mathrm{c}^{2}$ for the SM not to be trivial [2]. Direct searches at LEP provided a limit $m_{H}>114 \mathrm{GeV} / \mathrm{c}^{2}$ at $95 \%$ confidence level (CL) [3]. From a fit to the electroweak data [4] an upper limit has been set: $m_{H}<144 \mathrm{GeV} / \mathrm{c}^{2}$ at $95 \% \mathrm{CL}$, which goes up to $182 \mathrm{GeV} / \mathrm{c}^{2}$ if the direct search limit is included.

The Large Hadron Collider (LHC) constitutes the new hope for the discovery of the Higgs boson. The energy range it explores allow to perform inclusive searches covering all the mass range where the SM Higgs boson is expected. At the LHC, the $\mathrm{H}$ boson will be produced mainly via the gluon gluon fusion process. The vector boson fusions (VBF) has a cross section one order of magnitude smaller. Associated production with a vector boson or $t \bar{t}$ are also possible but less probable [5]. The $H \rightarrow \gamma \gamma, H \rightarrow Z Z^{*}$ and $H \rightarrow W W$ inclusive analysis here presented make use of Monte Carlo simulations where theoretical calculations up to the next to leading order (NLO) for both signal and background have been taken into account. The detector is simulated according to the present knowledge of its geometry and response. Details of the analysis are given in [6].

\section{THE $H \rightarrow \gamma \gamma$ CHANNEL}

In the low mass region, $m_{H}<140 \mathrm{GeV} / \mathrm{c}^{2}$, the $H \rightarrow \gamma \gamma$ is one of the most promising decay channel. Identification of photons, which are detected as clusters in the electromagnetic calorimeter, is crucial. Converted photons have a dedicated selection which make use of tracker informations in order to correctly reconstruct their energy. The trigger requires two isolated electromagnetic cluster of

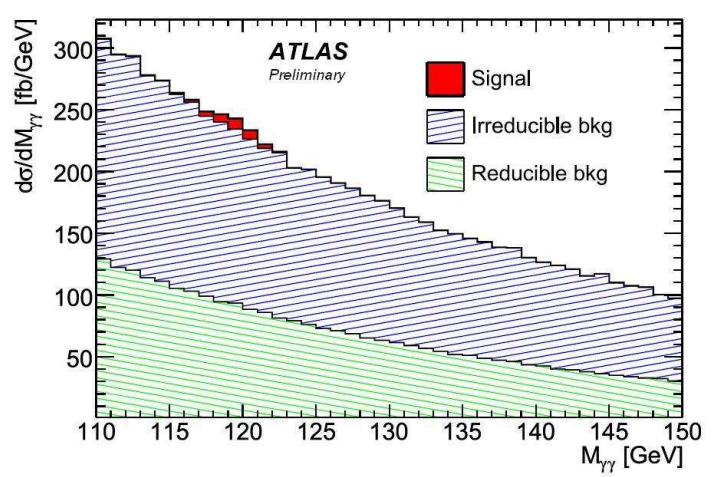

FIGURE 1. Two photons invariant mass distribution for $H \rightarrow$ $\gamma \gamma$.

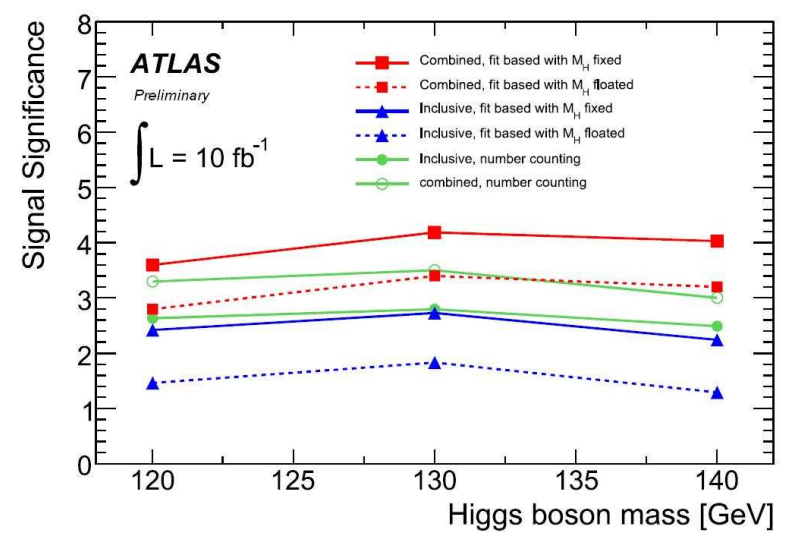

FIGURE 2. Sensitivity for $H \rightarrow \gamma \gamma$ in the inclusive and combined approaches, using number counting or a fit with fixed or floated Higgs mass.

transverse momentum greater than $20 \mathrm{GeV} / \mathrm{c}$, with an efficiency of $94 \%$ normalized to offline selections. A fiducial cut is applied to remove photon candidates in the region of pseudo-rapidity $1.37<\eta<1.52$, where for geo- 
metrical reasons the identification is not good. There are two main background components: the reducible background and the irreducible one.

The reducible background is mainly due to jets faking photons. A dedicated selection has been elaborated, exploiting the differences in the shower shapes of a jet and a photon. For a jet the presence of a second energy maximum in the electromagnetic cluster is expected and can be detected thanks to the fine segmentation of the first layer of the electromagnetic calorimeter. Jets will also deposit part of the energy in the hadronic calorimeter. Moreover track isolation is required: the sum of the transverse momentum $p_{T}$ of all tracks in a $\Delta R=0.3$ cone, where $\Delta R$ is the radial distance between the track and the cluster position, is computed for all tracks with $p_{T}>1$ $\mathrm{GeV} / \mathrm{c}$ and it is required to be lower than $4 \mathrm{GeV} / \mathrm{c}$. Conversion tracks are opportunely removed in this calculation. The two most energetic photon candidates are selected and they are required to have a $p_{T}$ greater than 25 and $40 \mathrm{GeV} / \mathrm{c}$ respectively.

The irreducible background comes from the direct production of photons. The only way to control it is by using some discriminating variables. The most powerful one is the invariant mass of the two photon candidates, which looks like a decreasing exponential for the background and a gaussian for the signal. The mass resolution is improved with a dedicated primary vertex reconstruction, combining in a likelihood the informations on the position of the clusters in the electromagnetic calorimeter and the direction of all the tracks of the event. Converted tracks, if reconstructed, are also properly taken into account. Figure 2 shows the two photon invariant mass distribution $m_{\gamma \gamma}$ with the different background and signal component after selection. Another interesting kinematic variable is the transverse momentum of the photons pair, which in the case of background have a softer spectrum with a less pronounced rise at low values. The module $\left|\cos \left(\theta^{*}\right)\right|$ of the cosine of the angle between one photon and the $p_{T}$ of the Higgs candidate momentum in the Higgs rest frame is also used: its distribution is flat for signal, being the Higgs a scalar vector, while tends to peak at 1 for backgrounds, even if the shape is smoothed by the detector acceptance in that region.

The analysis sensitivity is determined both using only the $m_{\gamma \gamma}$ variable (inclusive approach) and combining it with $p_{T}$ and $\left|\cos \left(\theta^{*}\right)\right|$ in an extended maximum likelihood fit (combined approach), leaving fixed or floating the Higgs mass. Results are shown on figure 2 and can be compared with a simple counting experiment approach. In the combined approach improvements are obtained when events belonging to different detector regions and coming from different topologies (associated production with one ore more jets) are classified in categories with different parameterizations of the discrimi-

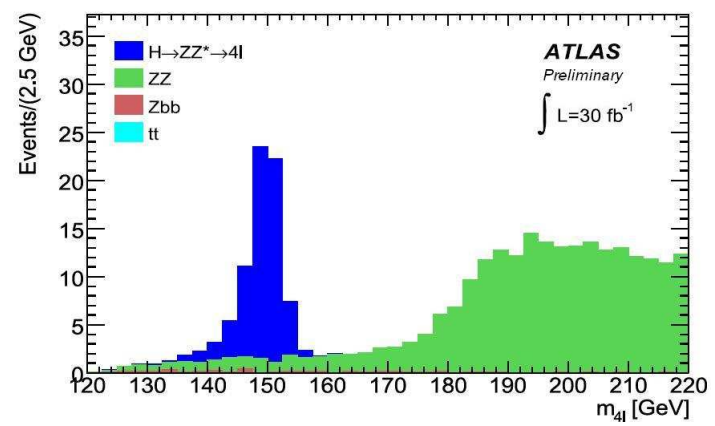

FIGURE 3. Four leptons invariant mass distribution for the mass hypothesis $150 \mathrm{GeV} / \mathrm{c}^{2}$

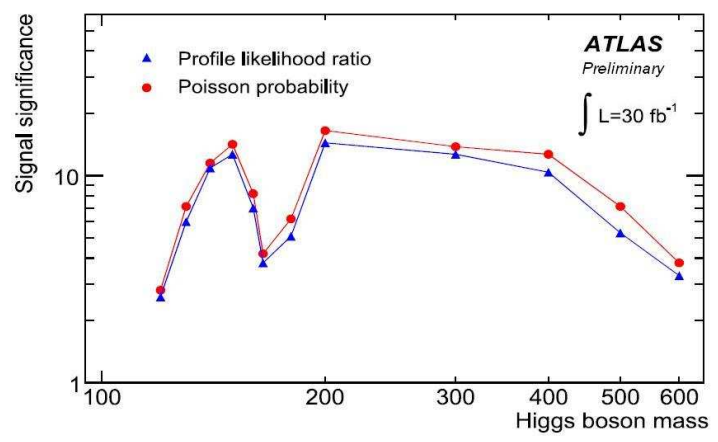

FIGURE 4. Sensitivity for the $H \rightarrow Z Z^{*}$ channel: red dots assume that the background is exactly known while blue triangles come from a fit with floated background normalization and shape parameters.

nating variables distributions.

\section{THE $H \rightarrow Z Z^{*}$ CHANNEL}

The $H \rightarrow Z Z^{*}$ channel starts to play an important role when $m_{H}>130 \mathrm{GeV} / \mathrm{c}^{2}$. This analysis scans the largest mass range, up to $600 \mathrm{GeV} / \mathrm{c}^{2}$ and it is certainly the one giving the cleanest signature, with a clear peak over a smooth background in the four leptons invariant mass (see Fig. 3). The crucial point here is having a very good identification of the electrons and muons coming from the $\mathrm{Z}$ decay. Electrons candidates have a cluster in the electromagnetic calorimeter and an associated track reconstructed from the inner detector. They can not have an associated energy deposit in the hadronic calorimeter and their shower shape should not be compatible with the hadron hypothesis. The muon identification is mainly based on the muon spectrometer but combines also inner detector and calorimeter informations. The trigger selects single electrons (muons) with $p_{T}>22(20) \mathrm{GeV} / \mathrm{c}$ or two electrons (muons) with $p_{T}>15(10) \mathrm{GeV} / \mathrm{c}$. Isolation of electrons is required. The kinematic of the event 
is exploited selecting two pairs of same flavor opposite charge leptons passing the fiducial cut and requiring that the invariant mass of one lepton pair lies in a $\Delta m_{12}$ window around the $Z$ mass and the invariant mass of the other pair is greater than a value $m_{34}$ (as an example, for $m_{H}=130 \mathrm{GeV} / \mathrm{c}^{2}, \Delta m_{12}=15 \mathrm{GeV} / \mathrm{c}^{2}$ and $m_{34}=20 \mathrm{GeV} / \mathrm{c}^{2}$ ).

Backgrounds can be estimated directly on data sidebands, giving a small systematic uncertainty. The main background sources are leptons from the decay of a $b$ quark coming for example from the process $q q \rightarrow Z b b$. This is strongly reduced by the isolation cut based on the calorimeter and the tracker, requiring $\Sigma p_{T} / p_{T}<0.15$ and in addition $\Sigma E_{T} / p_{T}<0.23$ for muons. Here $E_{T}$ is the transverse energy in a cone of $\Delta R=0.2$ around the muon direction and $p_{T}$ is the lepton transverse momentum. Moreover, since the $b$ quarks flight before decaying, the leptons they produce will not come from the primary vertex: for this reason a cut on the transverse impact parameter of the lepton is applied. Cuts are optimized depending on the final state: four electrons, four muons or two electrons and two muons. After these selections, the main surviving background is the irreducible one coming from $Z Z^{*}$ decays.

The significance of this channel has been estimated by a simple cut and count experiment as well as from different fit approaches and it is shown on figure 4.

\section{THE $H \rightarrow W W$ CHANNEL}

For $2 m_{W}<m_{H}<2 m_{Z}$, the dominant decay mode for the Higgs is a couple of $W$ bosons. The final state is made up of two charged leptons and two neutrinos which are responsible for the presence of missing energy in the event. The invariant mass distribution do not present any clear peak: the discriminating variable in this case is the transverse mass $m_{T}$. Two channels are explored: the final state $H \rightarrow W W \rightarrow e v_{e} \mu v_{\mu}$ and the final state $H \rightarrow W W \rightarrow l v_{l} q \bar{q}$. The second one is interesting since it will possibly allow a measurement of the spin and the $\mathrm{CP}$ properties of the Higgs and it is only studied for $m_{H}=300$ $\mathrm{GeV} / \mathrm{c}^{2}$, when the Higgs is produced in association with two jets via VBF. The $H \rightarrow W W \rightarrow e v_{e} \mu v_{\mu}$ analysis is particularly challenging since many backgrounds have to be estimated: direct production of $W W, W t, q \bar{q}$ (with $q=t, b, c$ quark), $Z \rightarrow l l$ and QCD multi-jets. The transverse mass and the transverse momentum of the candidates are fit in two bins of the dilepton opening angle $\Delta \Phi$ in the transverse plane. Once accounted for the ratio of the background in the two regions, the signal and background is extracted in the signal region. In the VBF case, an additional requirement on topology can be used: two forward jets must be tagged, with a large $\eta$ separation, high $p_{T}$, and an high invariant mass $m_{j j}$ value.
Moreover no other jets are allowed in the region between the two jets. The significance of the $H \rightarrow W W$ channel, combined all the analyzed final states, will be shown on [6].

\section{CONCLUSIONS}

The inclusive Standard Model Higgs searches in the decays $H \rightarrow \gamma \gamma, H \rightarrow Z Z^{*}$ and $H \rightarrow W W$ with the ATLAS detector have been presented. The analysis have been performed on simulations taking into account corrections up to the next to leading order and considering the present knowledge of the detector performance. The preliminary expected signal significances for $H \rightarrow \gamma \gamma$ and $H \rightarrow Z Z^{*}$, obtained with both conservative and improved statistical methods, have been shown. More details on the analysis, including a combination of the different channels, will be soon released [6].

\section{ACKNOWLEDGMENTS}

I would like to thank all the ATLAS collaborators, in particular the Higgs working group. I am particularly grateful to Louis Fayard and Leandro Nisati for their useful comments and suggestions.

\section{REFERENCES}

1. F. Englert and R. Brout, Phys. Rev. Lett. 13 (1964), 321; P. W. Higgs Phys. Lett. B 12 (1964), 132; P. W. Higgs Phys. Rev. Lett. 13 (1964), 508.

2. T. Hambye and K. Riesselmann, arXiv:hep-ph/9708416.

3. LEP Higgs working group, Phys. Lett. B565 (2003) 61.

4. LEP Electroweak Working Group, http://lepewwg.web.cern.ch/LEPEWWG/

5. A. Djouadi, Phys.Rept.457: 1-216, 2008.

6. ATLAS Collaboration, Expected Performance of the ATLAS Experiment, Detector, Trigger and Physics, CERN-OPEN-2008-020, Geneva, 2008, to appear. 